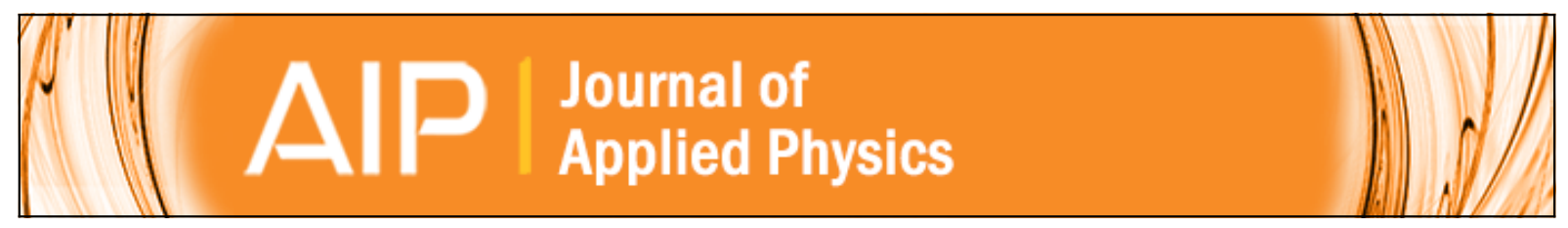

\title{
Effect of growth interruption on optical properties of In-rich In Ga N Ga N single quantum well structures
}

Yuanping Sun, Yong-Hoon Cho, H. M. Kim, T. W. Kang, S. Y. Kwon, and E. Yoon

Citation: Journal of Applied Physics 100, 043520 (2006); doi: 10.1063/1.2220514

View online: http://dx.doi.org/10.1063/1.2220514

View Table of Contents: http://scitation.aip.org/content/aip/journal/jap/100/4?ver=pdfcov

Published by the AIP Publishing

\section{Articles you may be interested in}

Correlating exciton localization with compositional fluctuations in In Ga N Ga N quantum wells grown on GaN planar surfaces and facets of GaN triangular prisms

J. Appl. Phys. 102, 093502 (2007); 10.1063/1.2802291

Radiative and nonradiative lifetimes in nonpolar m -plane In x Ga 1 x N Ga N multiple quantum wells grown on GaN templates prepared by lateral epitaxial overgrowth

J. Vac. Sci. Technol. B 25, 1524 (2007); 10.1116/1.2746354

Optical characteristics of a -plane In Ga N Ga N multiple quantum wells with different well widths Appl. Phys. Lett. 90, 181122 (2007); 10.1063/1.2735935

In Ga N Ga N nanostripe grown on pattern sapphire by metal organic chemical vapor deposition Appl. Phys. Lett. 90, 013110 (2007); 10.1063/1.2430487

Quantum dot emission from site-controlled In Ga N Ga N micropyramid arrays

Appl. Phys. Lett. 85, 4281 (2004); 10.1063/1.1815043

A|P| Applied Physics

A|P | Letters

is pleased to announce Reuben Collins as its new Editor-in-Chief

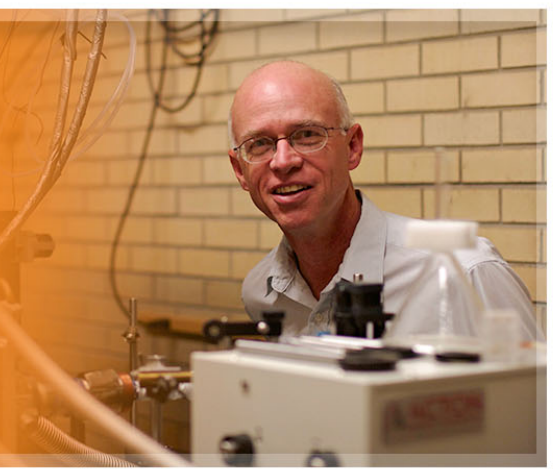




\title{
Effect of growth interruption on optical properties of In-rich InGaN/GaN single quantum well structures
}

\author{
Yuanping Sun \\ Institute of Science and Technology for Opto-electronic Information, Yantai University, Yantai 264005, \\ People's Republic of China; Department of Physics, Chungbuk National University, \\ Cheongju 361-763, Korea; and Institute for Basic Science Research, Chungbuk National University, \\ Cheongju 361-763, Korea \\ Yong-Hoon $\mathrm{Cho}^{\mathrm{a})}$ \\ Nano-Bio-Photonics Laboratory, Department of Physics, Chungbuk National University, \\ Cheongju 361-763, Korea and Institute for Basic Science Research, Chungbuk National University, \\ Cheongju 361-763, Korea \\ H. M. Kim and T. W. Kang \\ Quantum-functional Semiconductor Research Center, Dongguk University, Seoul 100-715, Korea
}

S. Y. Kwon and E. Yoon

School of Materials Science and Engineering, Seoul National University, Seoul 151-742, Korea

(Received 12 December 2005; accepted 27 May 2006; published online 29 August 2006)

In-rich InGaN/GaN single quantum well (SQW) structures with and without growth interruption (GI) were successfully grown on sapphire substrates by metal-organic chemical vapor deposition. The optical properties were systematically investigated by photoluminescence (PL), selectively excited PL, PL excitation (PLE), and cathodoluminescence (CL) techniques. The integrated PL intensity of the main In-rich InGaN emissions for the sample grown without GI decreased only by a factor of 15.5 when the temperature increased from 11 to $300 \mathrm{~K}$, while that of the sample with GI decreased by about 1040, showing very good quantum efficiency for the sample without GI. The In-rich InGaN SQW emissions have been verified by selectively excited PL spectra and by the different PLE absorption edges. CL observations showed that the epilayer of the sample without GI agglomerated together to form clusters due to the large lattice and thermal mismatches with GaN, which confine the carriers in the clusters and ensure the relatively high quantum efficiency of the sample. The sample with GI showed relatively smooth surface with cluster structures jointed together, which gives two-dimensional QW environment in its energy band structure, and its optical emission is more sensitive to temperatures than that of the sample grown without GI. (C) 2006 American Institute of Physics. [DOI: 10.1063/1.2220514]

\section{INTRODUCTION}

III-nitride semiconductor materials, including AlN, AlGaN, GaN, and InGaN, etc., have been widely studied ${ }^{1}$ and become the most promising materials for device applications of light emitting diodes and laser diodes ${ }^{2}$ in the wavelength range of ultraviolet $(\sim 6.2 \mathrm{eV}$ for AlN) to infrared $[\sim 0.7 \mathrm{eV}$ for InN Ref. 3]. As compared to the other III-nitride semiconductors, $\mathrm{InN}$ is rarely investigated because of the thermal instability as well as the large lattice and thermal mismatches, which make it very difficult to grow high quality epilayers. Recently, some advances have been reported for the growth of In-rich InGaN/GaN single quantum well $^{4}(\mathrm{SQW})$ as well as multiple quantum well (MQW) structures $^{5}$ by adopting growth interruption. In this paper, we report the optical properties of the In-rich InGaN/GaN SQW structures grown by metal-organic chemical vapor deposition (MOCVD) on sapphire substrates with and without growth interruption (GI). The detailed optical properties of samples

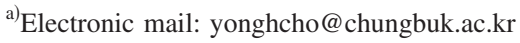

are investigated by temperature dependent photoluminescence (PL), selectively excited PL, PL excitation (PLE), and cathodoluminescence $(\mathrm{CL})$ techniques.

\section{EXPERIMENTS}

Two In-rich InGaN/GaN SQW structure samples were grown on $c$-axis sapphire substrates by MOCVD. The In-rich InGaN well layer was grown for $90 \mathrm{~s}$ at $730{ }^{\circ} \mathrm{C}$ on a 2 $-\mu \mathrm{m}$-thick GaN epilayer, and then a 20 -nm-thick GaN was grown as a capping layer at $730{ }^{\circ} \mathrm{C}$. The growth pressure was maintained at 300 Torr throughout the whole process. For the effective $\mathrm{NH}_{3}$ cracking at low growth temperatures ${ }^{6}$ and the reduction of native defects, ${ }^{7}$ the ammonia was preheated before it entered the reactor. During the growth of the well layer, only trimethylindium (TMIn) and ammonia were supplied as the sources of In and N, respectively. However, due to the solid-state intermixing of $\mathrm{InN}$ with underlying and capping GaN layers, the indium content of about $70 \%$ in the InGaN well layer was obtained by medium energy ion scattering (MEIS) experiments. ${ }^{8}$ No GI was introduced between the In-rich $\mathrm{InGaN}$ and $\mathrm{GaN}$ capping layers for sample A. Sample B was grown under the same condition as sample A 

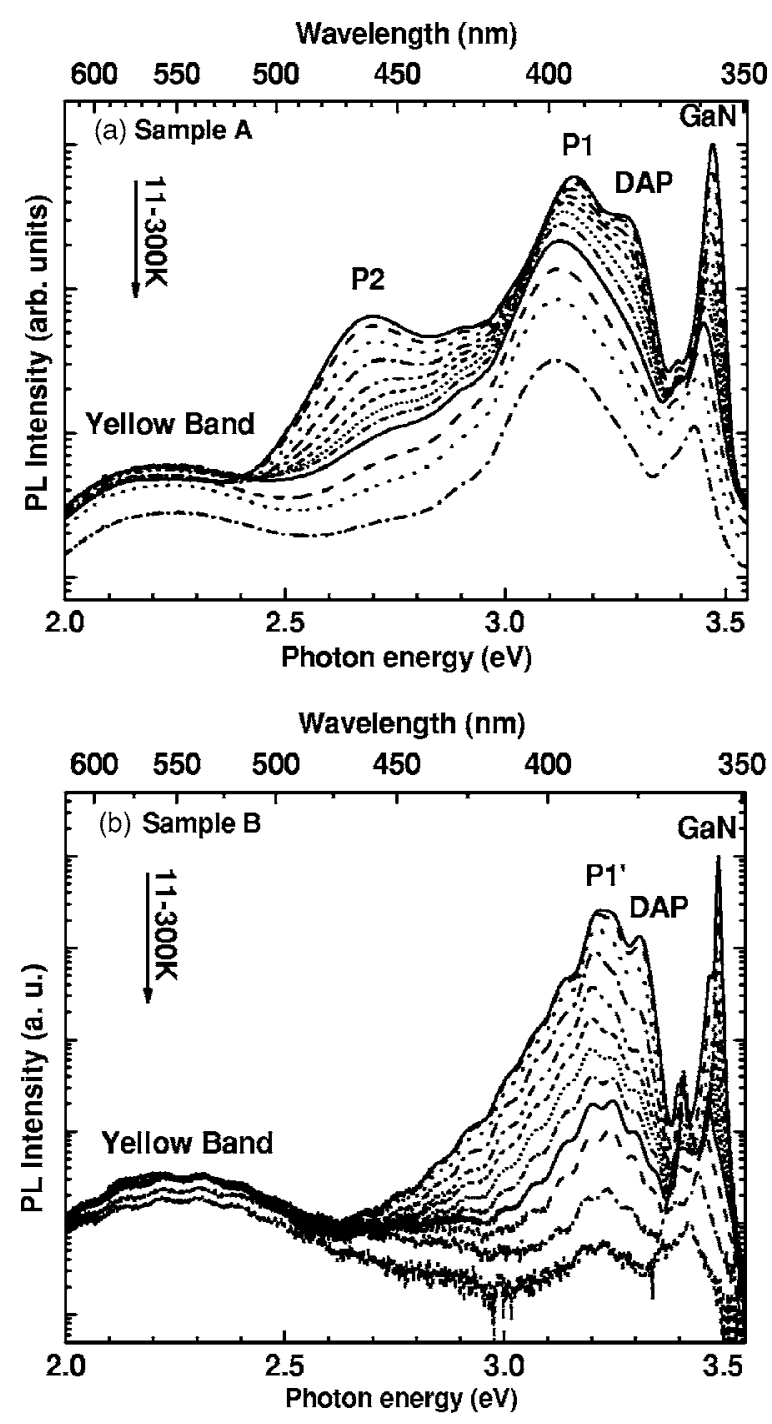

FIG. 1. Temperature dependent PL spectra of (a) sample A (without GI) and (b) sample B (with GI). Integrated PL intensity of the In-rich InGaN emission decreases by a factor of 15.5 and 1040 for samples A and B, respectively. The measured temperatures are 11, 30, 50, 70, 90, 110, 130, 150, 175, 200,250 , and $300 \mathrm{~K}$.

except for the $5 \mathrm{~s}$ GI after the growth of InN for the aim of a planar surface of the sample. The In-rich InGaN/GaN SQW structures were characterized by PL, PLE, and CL. A $\mathrm{He}-\mathrm{Cd}$ laser $(325 \mathrm{~nm})$ was used for the temperature dependent PL experiments with temperature ranging from 11 to $300 \mathrm{~K}$. A Xe lamp dispersed by a monochromator was used as a pumping source for the selectively excited PL and PLE experiments. CL spectra and CL images were acquired at $80 \mathrm{~K}$ by using a commercialized CL system with a highresolution scanning electron microscope with an electron accelerating voltage of $10 \mathrm{kV}$.

\section{RESULTS AND DISCUSSION}

Figure 1 shows temperature dependent PL spectra acquired from 11 to $300 \mathrm{~K}$ for sample A (without GI) and sample B (with $5 \mathrm{~s}$ GI after the growth of InN layer). The $\mathrm{GaN}$ band edge emission, the donor-to-acceptor pair (DAP) emission, and the yellow band emission peaks from the $\mathrm{GaN}$ layers are clearly seen for both samples. Two emission peaks (denoted as P1 and P2) for sample A and one main emission peak (denoted as P1') for sample B are also observed and originated from the In-rich InGaN SQW. The relatively high energy of the emission peak from In-rich InGaN SQW with the In composition of $\sim 70 \%$ can be attributed to the thin well width $(\sim 1 \mathrm{~nm})$ and the compositional grading due to the intermixing of $\mathrm{InN}$ and $\mathrm{GaN}^{8}$. The integrated intensity of the main emission peaks for sample A decreased by a factor of only 15.5 with increasing temperature from 11 to $300 \mathrm{~K}$, reflecting a very good quantum efficiency even at high temperature, while that for sample B decreased by a factor of 1040 for the same temperature increase. The only difference between the two samples during the growth process is the $5 \mathrm{~s}$ GI after the growth of InN layers for sample B, which aimed to smooth the sample surface through the adatom migration. However, it seems that cluster structures are vital to our Inrich InGaN SQW samples to maintain its high-efficiency light emission at high temperature. As we have demonstrated for the trapezoidal InGaN MQW samples, the phase separation can localize the carriers in the potential fluctuations and decrease the probability to meet nonradiative center, which improve the light emission efficiency at high temperature greatly. ${ }^{9}$ Due to the GI of sample B the formation of cluster structures is depressed, and the integrated intensity of the main SQW emission for sample B (with GI) decreases more than that for sample A (without GI).

Thermal escape of carriers from well to barrier causes luminescence quenching for an ideal quantum well, in which the activation energy equal to the QW binding energy of electrons and/or holes. ${ }^{10}$ For real cases, however, there are always some nonradiative recombination centers in the QW with the active energy smaller than the QW binding energy of electrons and holes. ${ }^{11}$ If there are several kinds of nonradiative channels, the integrated PL intensity can be expressed by the following multichannel Arrhenius plot formula:

$$
I=I_{0} /\left[1+\sum_{i} C_{i} \exp \left(-E_{A i} / k_{B} T\right)\right],
$$

where $E_{A i}$ are the activation energies of the corresponding nonradiative recombination center and $C_{i}$ are the constants related to the density of these centers. Figure 2 shows the normalized integrated PL intensity of the main emission for samples A and B together with the best fitting of the Arrhenius plot. The best fitting gives the activation energies of 207 and $132 \mathrm{meV}$ for samples $\mathrm{A}$ and $\mathrm{B}$, respectively. The activation energies often referred as the localization energy of deep localized states, ${ }^{12}$ in which carriers can be trapped and have less chance to meet other nonrecombination centers.

To verify the origin of peaks P1 and P2 for sample A and P1' for sample B, selectively excited PL and PLE experiments are conducted at $11 \mathrm{~K}$ by using a Xe lamp dispersed by a monochromator as pumping source. Figure 3 shows the PL spectra excited at 325 and 387 (378) nm for sample A (B), at which the excitation energy is above and below the GaN band gap energy, respectively. Peaks P1 and P2 for sample A and peak $\mathrm{P}^{\prime}$ for sample B were still clearly seen when the samples were excited by the energy below the DAP peaks, indicating that the $\mathrm{P} 1, \mathrm{P} 2$, and $\mathrm{P} 1$ ' peaks are not related to $\mathrm{GaN}$ but to InGaN SQW. From the result, we can 


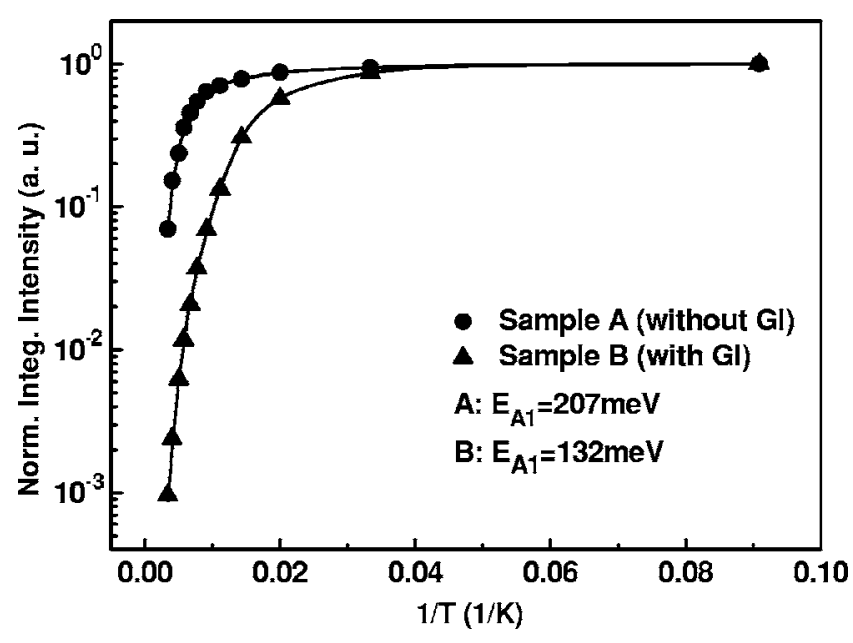

FIG. 2. Arrhenius plot of the In-rich InGaN emission for sample A (without GI) and sample B (with GI). The best fitting gives the activation energies of $207 \mathrm{meV}$ for sample A and $132 \mathrm{meV}$ for sample B.
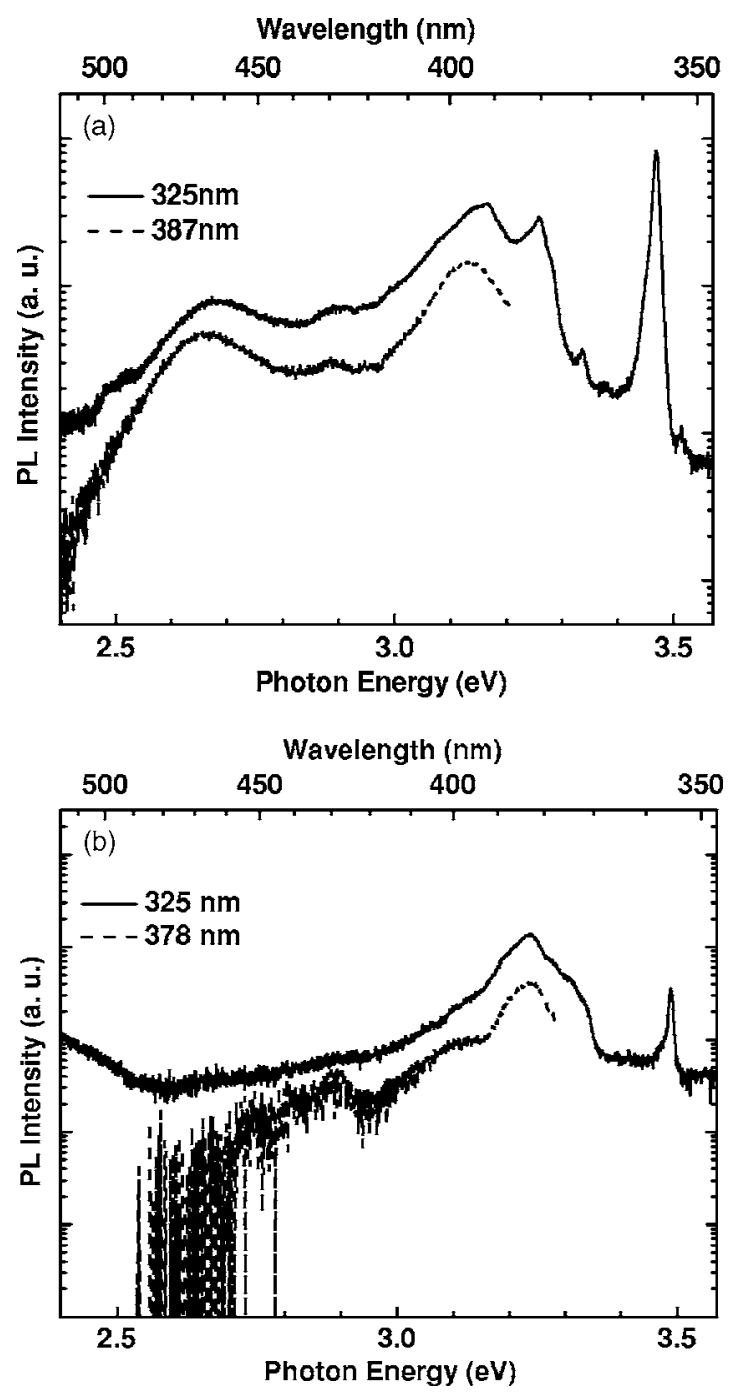

FIG. 3. Selectively excited PL spectra measured at $11 \mathrm{~K}$ using a Xe lamp excitation. The excitation wavelengths of 325 and 387 (378) nm are used for sample A (B), which are above and below the GaN band gap energy, respectively.
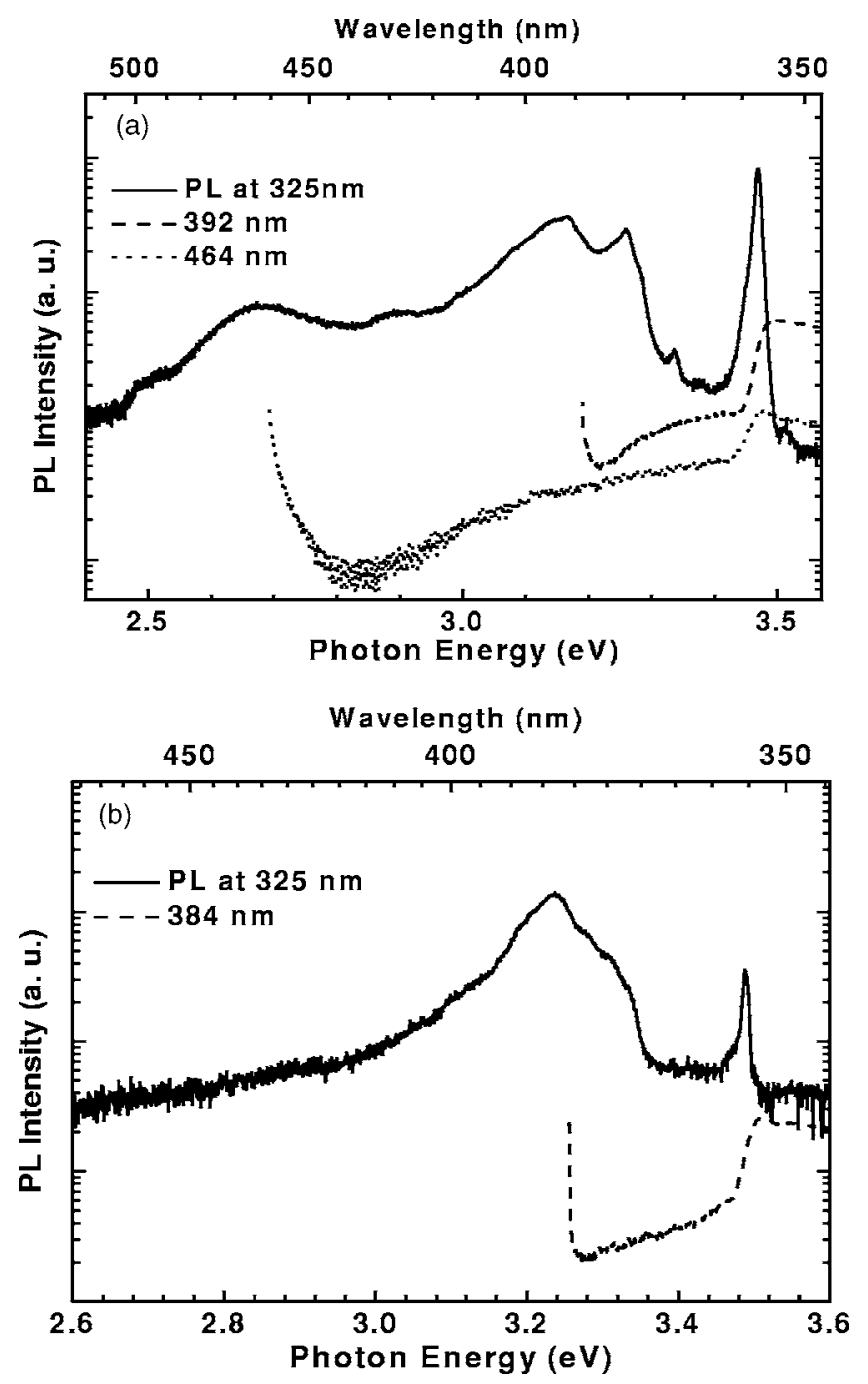

FIG. 4. PLE spectra measured at $11 \mathrm{~K}$ for (a) sample $\mathrm{A}$ (without GI) and (b) sample B (with GI). The detection energy was set at 392 and $464 \mathrm{~nm}$ for sample A and $378 \mathrm{~nm}$ for sample B.

assign these emission peaks to In-rich InGaN SQW regions. Figure 4 shows PLE spectra measured at $392 \mathrm{~nm}$ (P1) and $464 \mathrm{~nm}(\mathrm{P} 2)$ for sample A and $378 \mathrm{~nm}\left(\mathrm{P} 1^{\prime}\right)$ for sample B at $11 \mathrm{~K}$, together with the PL data excited at a wavelength of $325 \mathrm{~nm}$ from the same Xe lamp. Clear absorption edges are shown for GaN and In-rich InGaN layers for both samples. Large Stokes-like shifts of the In-rich InGaN emissions between the PL peak position and PLE absorption edge are observed. The PLE contribution of the GaN layer to the Inrich InGaN emissions can be clearly seen for all PLE spectra. However, for the PLE spectrum detected at $463.6 \mathrm{~nm}$ (P2), no specific PLE feature appears near the P1 peak, reflecting that the channel between the P1 and P2 emissions are not well connected to each other.

Figure 5 shows the CL spectrum and images taken at $356 \mathrm{~nm}(\mathrm{GaN}), 389.5 \mathrm{~nm}(\mathrm{P} 1)$, and $452.5 \mathrm{~nm}(\mathrm{P} 2)$ at $80 \mathrm{~K}$ for sample A. Figure 6 shows the CL spectrum and images taken at $353.6 \mathrm{~nm}(\mathrm{GaN})$ and $380.6 \mathrm{~nm}\left(\mathrm{P}^{\prime}\right)$ at $80 \mathrm{~K}$ for sample B. For sample A, the small cluster structures cover the full fields and all the emissions are from the cluster structures. Although the CL emission from GaN covers the full cluster together, peak P1 emitted from only the side edge of 


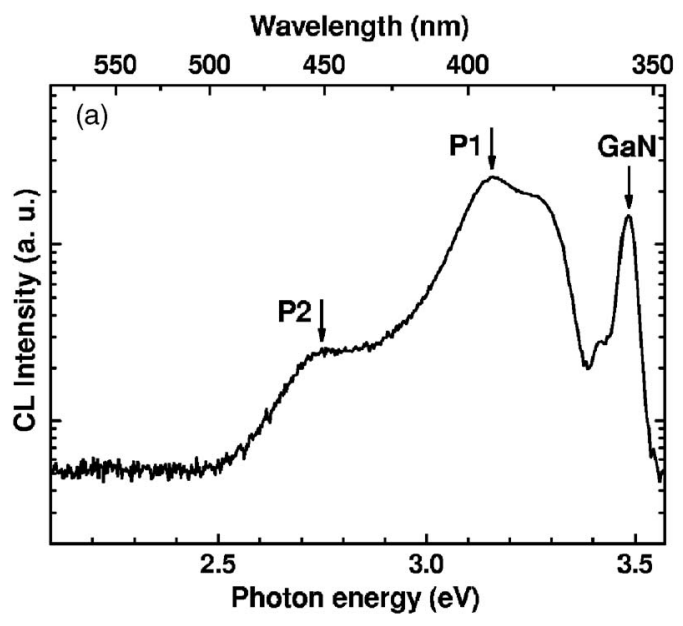

FIG. 5. CL spectrum and CL images measured at $80 \mathrm{~K}$ for sample A (without GI). (a) CL spectrum and [(b)(d)] CL images measured at (b) GaN, (c) P1, and (d) P2 [as denoted in (a)].

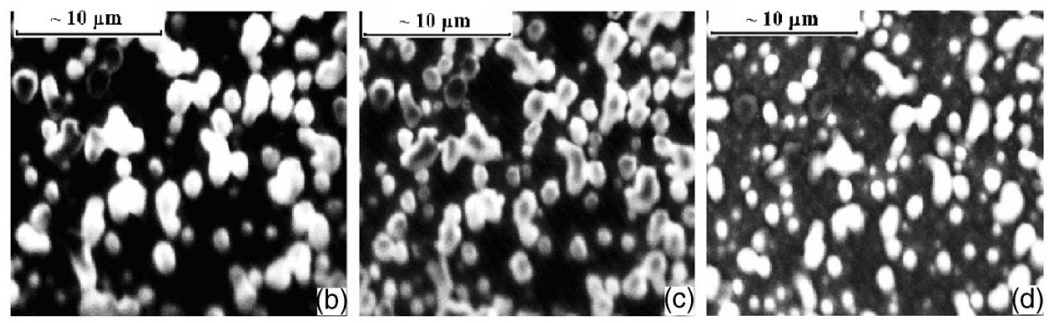

each cluster and peak P2 comes from the central part of each cluster, leading to $\mathrm{CL}$ images with reverse contrast. For sample $\mathrm{B}$, because of the adatom migration and severe decomposition during $\mathrm{GI}^{4}$, the surface becomes relatively smooth and the clusters have jointed together with clear conjunction borders. Both the CL images taken from GaN and P1' seem from the merged island structures together with
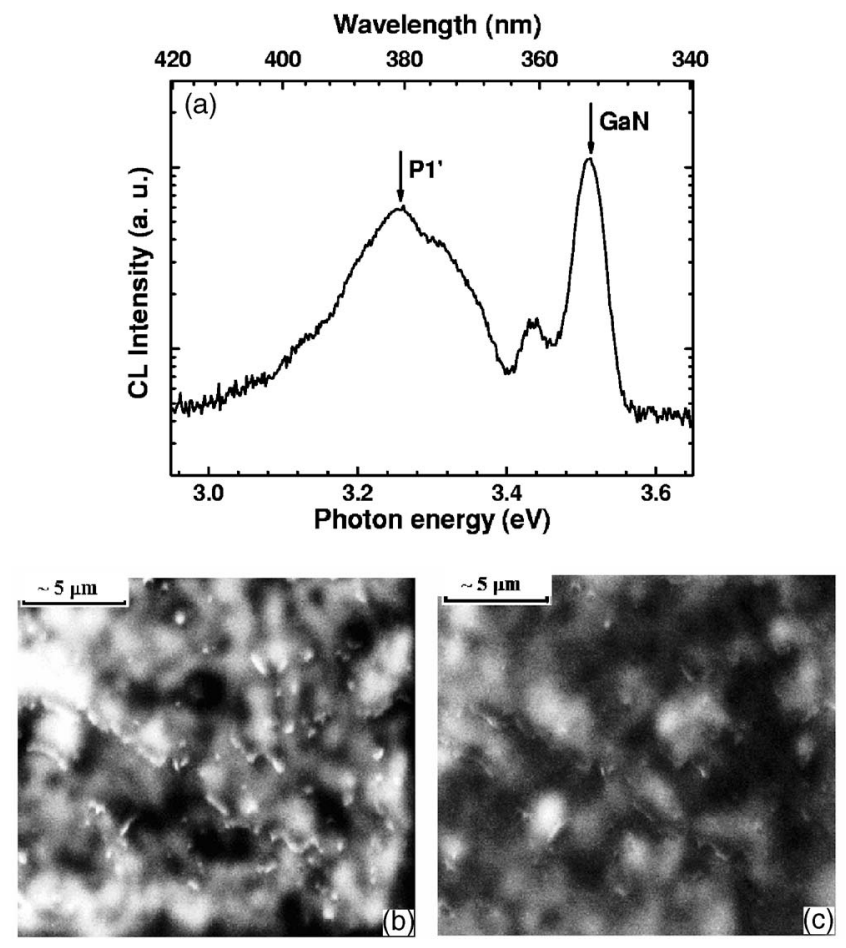

FIG. 6. CL spectrum and CL images measured at $80 \mathrm{~K}$ for sample B (with GI). (a) CL spectrum; and [(b)-(c)] CL images measured at (b) GaN and (c) $\mathrm{P}^{\prime}$ [as denoted in (a)]. some of the conjunction parts of the islands. From the results, the growth of planar InN layer may be very difficult without GI (sample A), because it is inclined to agglomerate together to form clusters due to the large lattice and thermal mismatches between $\mathrm{GaN}$ and $\mathrm{InN}$. The GaN layers deposited before and after the InN layer may package it inside to form the clusters. More $\mathrm{GaN}$ is included in $\mathrm{InN}$ region at the side edge of the clusters, while the central part of the clusters is possessed of less amount of $\mathrm{GaN}$, resulting in different $\mathrm{CL}$ (PL) for P1 and P2 of sample A. If GI was introduced to smooth the surface (sample B), these clusters will joint together to form a relatively smooth surface that gives twodimensional (2D) QW environment in its energy band structure, which will increase the probability of carriers' meeting with nonradiative centers and decrease quantum efficiency (as shown in Fig. 2).

\section{CONCLUSIONS}

The In-rich InGaN/GaN SQW samples were grown by MOCVD on sapphire substrate with and without GI. Optical properties were studied in detail by temperature dependent PL, selectively excited PL, PLE, and CL. Higher quantum efficiency of the emission was found for the sample without GI than that with GI. The In-rich InGaN SQW emissions have been verified by selectively excited PL spectra and by the different PLE absorption edges. CL observation showed that cluster structures are formed for the sample grown without GI, while the sample grown with GI forms a relatively smooth surface by the joints of clusters which gives a twodimensional QW environment in its energy band structure. This explains why the emission intensity of the sample with GI more sensitively decreases with increasing temperature than that of the sample without GI. 


\section{ACKNOWLEDGMENTS}

This work was supported by the Korea Science and Engineering Foundation through the National Research Laboratory Program and through the QSRC at Dongguk University One of the authors (Y.S.) acknowledges the support from the National Natural Science Foundation of China with Grant No. 10404022.

${ }^{1}$ I. Akasaki, H. Amano, Y. Koide, K. Hiramnatsu, and N. Sawaki, J. Cryst. Growth 98, 209 (1989).

${ }^{2}$ S. Nakamura, S. Pearton, and G. Fasol, The Blue Laser Diode (Springer, Berlin, 2000).
${ }^{3}$ M. Yoshimoto, H. Yamamoto, W. Huang, H. Harima, J. Saraie, A. Chayahara, and Y. Horino, Appl. Phys. Lett. 83, 3480 (2003).

${ }^{4}$ S.-Y. Kwon et al., Phys. Status Solidi C 0, 2830 (2003).

${ }^{5}$ S.-Y. Kwon et al., Appl. Phys. Lett. 86, 192105 (2005).

${ }^{6}$ H. J. Kim et al., Curr. Appl. Phys. 3, 351 (2003).

${ }^{7}$ S.-Y. Kwon, H. J. Kim, B. Kee, H. Na, and E. Yoon, Phys. Status Solidi C 0, 405 (2002).

${ }^{8}$ S.-Y. Kwon et al., Phys. Status Solidi A 201, 2818 (2004).

${ }^{9}$ Y. Sun, Y.-H. Cho, E.-K. Suh, H. J. Lee, R. J. Choi, and Y. B. Hahn, Appl. Phys. Lett. 84, 49 (2004).

${ }^{10}$ S. Weber, W. Limmer, K. Thonke, P. Sauser, K. Panzlaff, G. Bacher, H. P. Meier, and P. Roentgen, Phys. Rev. B 52, 14739 (1995).

${ }^{11}$ Y. Narukawa, Y. Kawakami, S. Fujita, and S. Nakamura, Phys. Rev. B 59, 10283 (1999).

${ }^{12}$ S. Nakamura, Mater. Sci. Eng., B 50, 272 (1997). 